\title{
Configural Response Learning: The Acquisition of a Nonpredictive Motor Skill
}

\author{
Eliot Hazeltine \\ University of Iowa
}

\author{
Paul Aparicio \\ Massachusetts Institute of Technology
}

\author{
Andrea Weinstein and Richard B. Ivry \\ University of California, Berkeley
}

\begin{abstract}
This study examined the representational nature of configural response learning using a task that required simultaneous keypresses with 2 or 3 fingers, similar to the production of chords on the piano. If the benefits of learning are related to the retrieval of individual stimulus-response mappings, performance should depend on the frequencies of the individual responses forming each chord. Alternatively, learning may involve the encoding of configural information concerning the relationship between the chord elements. In Experiment 1, training was restricted to a subset of the 120 possible 3 -element chords. Probe blocks included the practiced chords, chords composed of novel configurations of practiced elements (reconfigured), and chords that contained a new element (new). Practiced chords were performed faster than reconfigured chords, indicating learning involves the encoding of configural information. Experiment 2 showed that learning was not restricted to configurations within each hand. Experiments 3 and 4 demonstrated that learning was largely response based.
\end{abstract}

Keywords: skill learning, bimanual control, implicit learning, motor learning, response selection

Humans possess a remarkable ability to acquire new motor skills. Motor skill learning has traditionally been studied with experimental tasks such as the serial reaction time (SRT) task (Nissen \& Bullemer, 1987), rotary pursuit (Ammons, Ammons, \& Morgan, 1958), visuomotor transformations (Martin, Keating, Goodkin, Bastian, \& Thach, 1996; Welch, 1978), rhythmic interlimb coordination tasks (Amazeen, 2002; Newell, Liu, \& MayerKress, 2001; Zanone \& Kelso, 1997), and, more recently, force field adaptation (Shadmehr \& Mussa-Ivaldi, 1994). A notable feature of these tasks is their emphasis on how people learn to chain together a series of gestures into a coherent action. For example, rotary pursuit and the SRT task were specifically designed to look at sequence learning, with behavioral studies focusing on the form of representation underlying sequential representations (A. Cohen, Ivry, \& Keele, 1990; Hazeltine, Teague, \& Ivry, 2002; Keele, Jennings, Jones, Caulton, \& Cohen, 1995; Willingham, 1998; Willingham, Wells, Farrell, \& Stemwedel, 2000); and brain imaging studies exploring the neural circuits that show learning-related changes as people come to anticipate future events (for reviews, see Keele, Ivry, Mayr, Hazeltine, \& Heuer, 2003; Ungerleider, Doyon, \& Karni, 2002).

Eliot Hazeltine, Department of Psychology, University of Iowa; Paul Aparicio, Department of Brain and Cognitive Sciences, Massachusetts Institute of Technology; Andrea Weinstein and Richard B. Ivry, Department of Psychology, University of California, Berkeley.

This work was supported by National Institutes of Health Grants NS33504 and NS30256.

Correspondence concerning this article should be addressed to Eliot Hazeltine, Department of Psychology, University of Iowa, 11 Seashore Hall E, Iowa City, IA 52242-1407. E-mail: eliot-hazeltine@uiowa.edu
Many manual skills not only require the successful coordination of successive movements with different effectors but also require that the effectors be appropriately configured. This point is made clear by the emphasis instructors place when teaching a wide range of skills. For example, golfers must learn to grip their clubs with the proper orientation and overlapping of the two hands, with the optimal configuration varying across different types of strokes. Similarly, when learning to type or to play musical instruments, such as the piano or violin, individuals often experience great difficulty in simultaneously producing the appropriate movements across multiple effectors.

To investigate configural response learning, we turned to a task introduced by Seibel (1963; see also Gopher, Karis, \& Koenig, 1985; Rabbitt, Fearnley, \& Vyas, 1975; Ratz \& Ritchie, 1961) in his classic study on response selection. In this task, the stimulus displays consisted of 10 lights, each of which was mapped to 10 response keys with a spatially compatible stimulus-response (S-R) mapping. On a trial, any combination of the 10 lights could be illuminated, and the participants were instructed to simultaneously press the corresponding buttons. Thus, there were $2^{10}-1(1,023)$ possible stimulus possibilities, each associated with a unique chord-like response. During the 150 30-min sessions of practice (equivalent to 75 presentations of each chord), reaction times (RTs) decreased from well over $1 \mathrm{~s}$ to about $400 \mathrm{~ms}$.

After completing this training, participants performed sessions in which the possible responses were restricted to a single hand. Despite the dramatic change in the number of possible responses (only the 31 combinations that involved only the digits of the right hand), mean RTs were only $30 \mathrm{~ms}$ faster compared to those obtained when the full complement of 1,023 options was tested. Seibel (1963) interpreted these results as indicating that perfor- 
mance no longer obeyed the Hick-Hyman law (Hick, 1952; Hyman, 1953) describing the manner in which RT was linearly related to the number of response alternatives. Rather, Seibel suggested that groups of lights were chunked into higher-order representations, allowing participants to encode information with greater efficiency.

Unexplored in this seminal study is what information is encoded as participants learn to perform the task. Specifically, it is unclear whether the participants were encoding general features of the task or encoding information specific to individual chords. To illustrate this distinction, consider the manner in which learning is assessed in the SRT task (A. Cohen et al., 1990; Curran \& Keele, 1993; Nissen \& Bullemer, 1987). The preferred measure of learning is the difference in performance, measured in terms of RT, between trials in which the stimuli appear in a fixed sequence and trials in which the stimuli occur in a random order. This measure is adopted because it is recognized that overall improvements in RT may relate to learning that is not related to sequence knowledge; for example, performance should improve as participants become proficient in accessing stimulus-response (S-R) mappings on individual trials, independent of whether the successive events are sequential or random. Comparing performance between blocks in which the stimuli are selected at random with those in which they follow a sequence provides a specific measure of sequence learning.

The same distinction can be applied to configural response learning. On the one hand, improvements in the chord task could reflect the encoding of the specific movement combinations. A demanding component of the task is the production of the configural chord responses, many of which require the coactivation of novel finger combinations (MacKay, 1982). We use the term configural in a restricted sense here, referring to the coactivation of specific effectors or keypresses, not just the relative positions of the effectors or keypresses. That is, learning to produce a particular configuration (e.g., left ring finger, left index finger, and right middle finger) should not transfer to configurations with the same spacings but anchored to different fingers (e.g., left middle finger, left thumb, and right ring finger). If learning is based on this sort of configural information, the benefits of practice should not transfer to novel chord patterns. In this case, the task could be thought of as requiring the learning of novel postures restricted to the hands.

Alternatively, given the number of potential combinations, learning may not be restricted to the encoding of specific configural responses but rather might involve the encoding of properties of the task that generalize across all possible chords. For example, learning might center on improving the efficiency of processes involved in S-R translation, possibly because of chunking or the development of automatic S-R associations, as suggested by Seibel (1963). In this case, the specific configural properties of the response would not affect performance. Thus, practice on a subset of the chords would lead to improved performance on all chords.

Hazeltine et al. (2002), addressed a related question with a study in which participants simultaneously performed two tasks involving visual-manual and auditory-vocal S-R pairings. On each trial, participants received either a single visual stimulus requiring a manual response, a single auditory stimulus requiring a vocal response, or both a visual and an auditory stimulus, each requiring a response. Unbeknownst to the participants, certain pairings of visual and auditory stimuli were never presented during training. When these withheld pairs were introduced after several sessions of training, they were performed as quickly and accurately as practiced combinations, suggesting that learning under these conditions did not involve encoding specific combinations of stimuli (or responses). Instead, participants learned to perform the two tasks independently, and performance did not depend on the particular S-R combinations.

However, it is an open question whether this result would have been obtained had both tasks used the same stimulus modality and effector system. In many learning tasks, there is strong evidence that specific items are encoded during training. For example, in the verbal-learning literature, there is clear evidence from a range of experimental tasks that repeated stimuli are categorized more rapidly than novel stimuli (N. J. Cohen \& Squire, 1980; Kirsner \& Speelman, 1996; Poldrack \& Gabrieli, 2001; Schwartz \& Hashtroudi, 1991). This pattern suggests that learning entails more than just the acquisition of a new skill; information about the particular stimuli encountered during training is also encoded. Moreover, this phenomenon, termed repetition priming, is not restricted to verbal tasks (Gabrieli, Milberg, Keane, \& Corkin, 1990; Poldrack, Selco, Field, \& Cohen, 1999).

Finally, it is possible that learning could occur at multiple levels: with training, people could become better at generic aspects of the task, such as efficient S-R selection for individual finger movements, as well as the production of specific configural responses. As demonstrated by Gupta and Cohen (2002), both of these types of information might be encoded by a common set of mechanisms (see also Logan, 1990; Poldrack et al., 1999).

In sum, whereas sequence learning involves encoding relationships between successive events, chord learning reflects the development of fluency in the production of multidigit movements. This type of learning may be specific to particular configural responses or it may relate to the ability to coordinate simultaneous responses regardless of whether the responses had been previously produced together. The following experiments explore this question.

\section{Experiment 1}

We employed a modified version of Seibel's (1963) task to study configural learning. On each trial, stimuli were presented at 3 of 10 possible locations and participants were instructed to simultaneously press the corresponding three keys, in a fashion similar to the way pianists play chords. A limited subset of the possible three-finger chords was practiced during training. This set of practice chords was arranged such that 1 of the 10 fingers was never used. At transfer, all of the possible chords were tested. We compared performance on three types of trials: trials in which the produced chord had been practiced (old chords), trials in which the chord was unpracticed but all of the component responses were practiced during training (reconfigured chords), and trials in which the chord included the withheld finger (new chords).

If learning in the chord task is primarily related to encoding specific response combinations (i.e., chord-level learning), then both the reconfigured and new chords should be performed more slowly than the old chords. In contrast, if learning is related to improvements in S-R translation for the individual elements that define a chord, then the old and reconfigured chords should be 
performed faster than the new chords; indeed, if learning is restricted to the element level, performance should be similar for old and reconfigured chords. This prediction holds because the old and reconfigured chords comprise identical sets of S-R associations at the element level. The two types of stimuli differ only in terms of the configural properties. Finally, if learning occurs at a more abstract level associated with general features of the task (S-R translation, combining elements into configural patterns), performance should be similar across all three conditions at transfer.

The extensive training provided by Seibel (1963) lasted several months. In the present study, we examined learning within a single session of practice. Although this dramatic difference in exposure to the task may affect the nature of the learning, our goal was to explore configural learning under conditions similar to those that have been used in studies of sequence learning.

\section{Method}

\section{Participants}

Ten participants were recruited from the undergraduate population at the University of California, Berkeley. The participants received monetary compensation with bonuses for high levels of performance. All participants agreed to participate in the study in accordance with the guidelines of the University's Committee for the Protection of Human Subjects.

\section{Apparatus and Stimuli}

Participants responded manually to visual stimuli presented on a computer screen. Responses were made on two 5-key response boards $(20 \mathrm{~cm} \times 30 \mathrm{~cm})$ with spatially compatible piano style keys ( $2 \mathrm{~cm}$ wide, $10 \mathrm{~cm}$ long for the four fingers and $13 \mathrm{~cm}$ long for the thumb). Participants adjusted the position of the response boards so that they could comfortably place all 10 fingers on the keys. The response boards were connected to a personal computer via the parallel port. A DOS-based program with millisecond accuracy was used to control the timing of the stimuli and sampling of the response devices.

Throughout the experiment, a row of 10 horizontal lines was displayed on the computer monitor, grouped into five lines on the left and five lines on the right. Each line was $1.1 \mathrm{~cm}$ in length and distance between lines within a side was $2.2 \mathrm{~cm}$. The distance between the two groups of lines was $4.9 \mathrm{~cm}$. Each of the horizontal lines was mapped to 1 of 10 keys on the two keyboards in a spatially compatible fashion.

There are 120 combinations of three-element configurations given 10 possible positions. However, one position, selected pseudorandomly from four possible positions, was excluded for each participant. The excluded position was always associated with either the index or ring finger of the right or left hand. This position is part of 36 configurations of the 120 chord set, leaving a total of 84 configurations formed by the full three-element combination of the remaining nine positions. Of these 84 configurations, 42 were designated to constitute the training set and the other 42 were reserved for the probe set, which was withheld until the final two blocks of the experiment. The two sets were matched in terms of the frequency with which each position appeared as well as other factors, such as the number of chords in which all three elements were from the same side. Note that the probe set was not restricted to 42 chords; rather, it included all 120 configurations.

\section{Procedure}

The trial began with the presentation of the chord stimulus, signaled by the presentation of three filled circles $(0.6 \mathrm{~cm}$ in diameter) above three of the horizontal lines. Subjects were instructed to make speeded responses by simultaneously pressing the three response keys that corresponded to the locations of the three circles. The chord stimulus was terminated after the response was completed, initiating a 1,500-ms intertrial interval. Errors were recorded when the participant failed to press the three correct keys or the responses were made sequentially, defined by trials in which all of the keys were released at some point between any two of the three keypresses. A $150 \mathrm{~ms}$ tone was played on incorrect trials prior to the start of the intertrial interval. In addition to the auditory online feedback, participants were given summary feedback of mean RT and total errors at the end of each block of trials.

Participants completed 10 blocks of trials. Only the training set was presented during the first eight blocks. Each of these blocks consisted of 126 trials, or three repetitions of each of the 42 training chords. The probe set was used on the last two blocks. These blocks consisted of 120 trials, or one presentation of each of the 120 chords (trained and untrained chords). Participants were permitted to take short breaks between the blocks as needed, but there was no additional break between the training and probe blocks; indeed, none of the participants reported being aware of any difference between the training and probe blocks.

Our main interest was in performance during the probe blocks. Here, we compared three types of chords: (a) 42 old chords consisting of the three-finger chords that had been practiced ( 24 times each) during the eight training blocks; (b) 42 reconfigured chords consisting of unpracticed chords in which each of the three elements had been included in the training set but not in these combinations; and (c) 36 new-finger chords consisting of the chords that included the element that had not been included in the training set.

\section{Results and Discussion}

\section{$R T s$}

For each trial, a single RT was recorded, taken as the maximum $\mathrm{RT}$ of the three component responses of a chord. RTs less than 250 $\mathrm{ms}$ or greater than $2,500 \mathrm{~ms}$ were eliminated from the analyses $(<$ $3 \%$ of trials). The average difference in onset times from first to last keypress on a trial was $30 \mathrm{~ms}$, and the difference was less than $50 \mathrm{~ms}$ on $88 \%$ of the trials, indicating that the participants followed the instructions to make the responses simultaneously. As a check, we recalculated the data using the alternative measure of the mean RT across the three component responses. The choice of measures did not alter the pattern of results.

RTs were submitted to two analyses, one to evaluate improvements in performance during the training period and one to evaluate transfer of learning. A linear regression testing for a decrease in the RTs from the training phase of the experiment revealed a significant linear decrease across the eight initial blocks, $F(1$, 
78) $=7.53, p<.01$. On the first block, the mean RT across participants was $1,407 \mathrm{~ms}$; on the eighth block, the mean RT was 1,155 ms (Figure 1). Thus, the participants exhibited considerable improvement on this task during the 50 min session.

Chord learning. Our primary focus was whether this learning would transfer to reconfigured and new-finger chord combinations. The analysis of variance (ANOVA) conducted on the probe phase of the experiment had two factors: block ( 9 vs. 10) and chord type (old, reconfigured, and new-finger). Performance on the probe blocks (Figure 1) produced significant main effects of block, $F(1,9)=5.22, p<$ .05 and chord type, $F(2,18)=7.31, p<.005$, and a significant interaction between the two factors, $F(2,18)=5.37, p<.05$. On Block 9, the first probe block, significant differences were observed between all three conditions. Old chords were responded to faster $(1,246 \mathrm{~ms})$ than reconfigured chords ${ }^{1}(1,313 \mathrm{~ms}), t(9)=2.77, p=$ .01 , which in turn elicited faster responses than to new-finger chords $(1,371 \mathrm{~ms}), t(9)=2.11, p<.05$. On Block 10, the differences between the probe types were smaller, with RTs for the old, reconfigured, and new-finger probes being 1,250, 1,279, and 1,307 ms, respectively. Only the difference between the old and new-finger probes was statistically reliable, $t(9)=1.93, p<.05$.

If learning was primarily the result of improved access to individual S-R mappings (see Van Selst, Ruthruff, \& Johnston, 1999), no differences should have been observed between the old and reconfigured chords. The results suggest otherwise: RTs were faster for old chords compared to reconfigured chords, even though the two conditions were composed of elements that had been practiced to a similar extent during training. Thus, individual chords were learned as specific configural responses during training. Although we refer to these as configural responses, configural learning could be in terms of improved pattern recognition (i.e., stimulus based), chord production (i.e., response based), or a combination of these two components. Unlike a piano, where each finger is used to press multiple keys and a single key may be pressed by different fingers, our configural learning task was constrained with single finger-key pairings. Given this constraint, we assume that response-based learning on this task is tied to specific effectors; that is, the participant learns to make a specific configural response rather than learns the relative spatial relationship between the selected keys, as often occurs during the playing of a piano. ${ }^{2}$

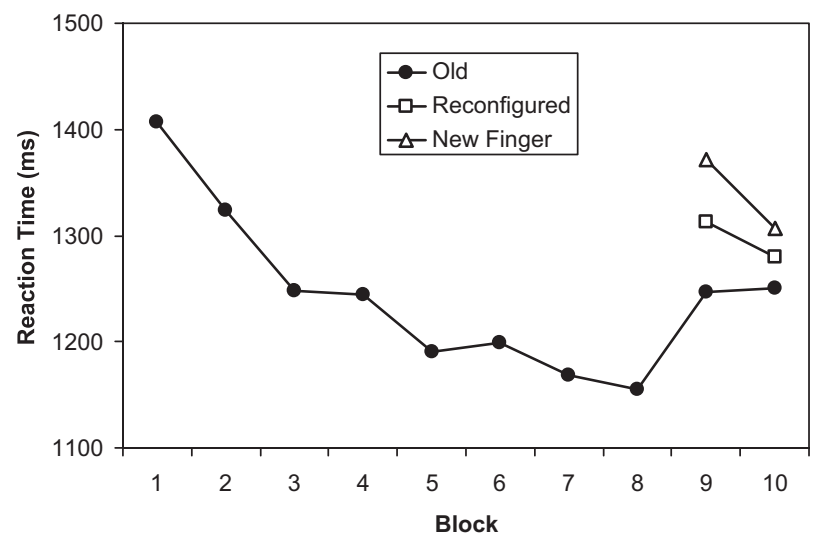

Figure 1. Reaction times in Experiment 1 across the 10 blocks for the three chord types, old, reconfigured, and new finger.
The new chords included a stimulus element and its associated response that had not been practiced. Thus, it was expected that these chords would be performed more slowly than old chords if the participants were learning specific configural responses. Although this prediction was confirmed, the new chords were also performed more slowly than reconfigured chords, indicating an additional cost associated with the introduction of a new element. This cost may indicate that learning not only occurs at a configural level but also at the element level. That is, the cost was due to the introduction of an unpracticed S-R mapping associated with the withheld finger. Alternatively, the increase in RT for the new chords may reflect the fact that the new chords were less similar to (i.e., contained fewer pairwise associations with) the practiced chords. A hypothesis to account for the increase in RT on the probe blocks is that the addition of a tenth finger increased the number of possible response combinations. The increase in RT here may be a manifestation of the Hick-Hyman law (Hick, 1952; Hyman, 1953) despite the fact that participants did not report being aware of the withheld finger during training. The present data do not provide a means to evaluate these hypotheses (see Gupta \& Cohen, 2002).

Sequential effects. An unexpected aspect of the results was the $91 \mathrm{~ms}$ increase in RT for the old chords when comparing the final training block and the first probe block. One methodological difference is that each of the old chords was presented three times in a training block and only once in a probe block. Therefore, exact repetitions of a chord are only possible in the training blocks. Perhaps the faster RTs for old chords on the last training block compared to the first probe block were due to the inclusion of repetition trials. Indeed, repetition RTs are faster by approximately $200 \mathrm{~ms}$ than nonrepetition RTs within the training blocks. However, even when we exclude repetition trials, the RTs for old chords on the probe blocks are approximately $80 \mathrm{~ms}$ slower than on the last training block. ${ }^{3}$

\footnotetext{
${ }^{1}$ Because the new and reconfigured chords were not expected to produce faster RTs than the old chords, one-tailed $t$ tests were used in all of the comparisons.

${ }^{2}$ To confirm that chord learning was anchored to specific effectors, we performed additional analyses on the data from the probe blocks. Some of the unpracticed chords were translated versions of practiced chords; but, for others, the spacings between the keypresses were unlike any of the chords in the training set (on average, each participant produced 21 such chords during the probe blocks). We therefore separated the unpracticed chords into two categories, translated and untranslated probes, and further divided these into new-finger and reconfigured chords. There was no indication that the untranslated chords were performed more slowly than translated chords (new finger: untranslated $1,330 \mathrm{~ms}$ vs. translated 1,370 $\mathrm{ms}, t<1$; reconfigured: untranslated $1,258 \mathrm{~ms}$ vs. translated $1,280 \mathrm{~ms}, t<$ 1). Thus, in the current task, configural learning appears to be anchored to the specific effectors rather than result from learning of abstract spatial configurations

${ }^{3}$ We also assessed whether the RTs for the three conditions depended on the condition of the previous trial. Given that this analysis involves nine cells ( 3 conditions on Trial $n$ crossed with the 3 conditions on Trial $n+1$ ), the data were combined across Blocks 9 and 10. The resulting ANOVA revealed a significant effect of chord, $F(2,18)=8.31, p<.005$, but no effect of previous chord, $F<1$, and no interaction, $F(4,36)=1.10, p>$ .3. This analysis indicates that the slower RT for old chords on the probe blocks was not due to a trial-by-trial carryover effect from the novel chords.
} 
We also assessed whether probe RTs were affected by similarity between successive chords. Each chord was scored in terms of how many of its elements were shared with the previous chord. For example, if on Trial $n+1$, the chord required pressing the second, fourth, and seventh keys and on Trial $\mathrm{n}$, the chord required pressing the first, fourth, and ninth keys, then the former trial was assigned an overlap score of 1 , because 1 key (the fourth) was involved in both chords. Trials with an overlap score of 3 are complete stimulus-response repetitions. In order to ensure that each cell of the analysis contained a reasonably large number of observations, we divided the training phase into two halves, with the first four blocks belonging to the early half and the remaining four belonging to the late half. The resulting ANOVA revealed a significant effect of training half, $F(1,9)=17.75, p<.005$, reflecting the shortening of RTs with practice, a significant effect of overlap, $F(3,27)=40.09, p<.0001$, and no interaction, $F(3$, $27)=1.99, p>.1$. These data are shown in Figure 2. Whereas the three levels of overlap for nonidentical chords produced RTs within $20 \mathrm{~ms}$ of each other, the exactly repeated chords were performed $200 \mathrm{~ms}$ faster. This finding is consistent with the proposal that the complex stimuli and movements comprising the chords are processed as a single entity; that is, learning is chord specific.

In sum, the analyses of sequential effects do not indicate why there was an increase in RT for the old chords on the probe blocks. ${ }^{4}$ The cost may reflect a general shift in strategy with the introduction of the novel chords: The presentation of these stimuli led to slower responses and this caused the participants to adopt a more conservative strategy for the entire probe blocks. As noted above, participants reported being unaware of the introduction of the novel chords. Thus, any change in strategy was implicit.

\section{Accuracy}

The proportions of correct responses were submitted to identical ANOVAs as the RTs. The ANOVA conducted on the data from the training phase of the experiment revealed a significant linear increase in accuracy across the 8 training blocks, $F(1,78)=9.05$,

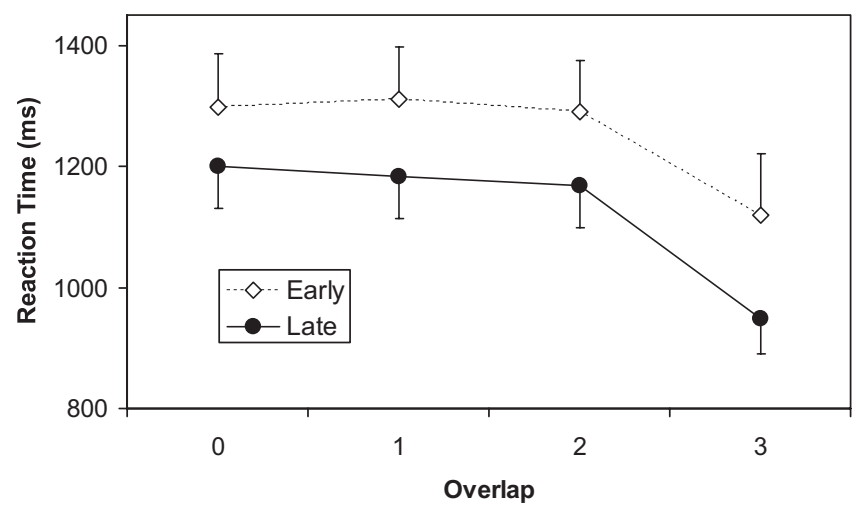

Figure 2. Sequential effects in Experiment 1 for chords based on the degree of overlap with the chord produced on the previous trial. The number of keypresses shared with the chord on the previous trial is indicated on the $x$-axis. Error bars represent 1 standard error of the mean. Early $=$ the first four blocks, Blocks $1-4$; Late $=$ the last four blocks of the training phase, Blocks 5-8. $p<.005$. On the first block, participants' mean proportion correct was .75; on the eight block, it was .86. There were no significant effects of block or chord type in the probe phase, although the pattern was similar to that observed in the RT data.

For the analysis of sequential effects, the patterns of accuracies were identical to those observed in the RTs. A reliable main effect of training half, $F(1,9)=7.74, p<.05$, indicated that participants became more accurate with practice $(.84$ correct on the first half; .87 correct on the second half). A reliable effect of overlap, $F(3$, $27)=5.83, p<.0001$, indicated that the nonoverlapping and partially overlapping trials were performed with mean accuracies ranging between .85 and .86 , whereas completely overlapping trials were performed with a mean accuracy of .91 . There was no evidence of an interaction, $F(3,27)<1$. Thus, speed-accuracy tradeoffs were not responsible for any of the effects observed in the RTs.

\section{Experiment 2}

The results of Experiment 1 demonstrate that improvement on the chord task results from configural learning. Had participants learned only individual S-R associations between single stimuli and keypresses, RTs on the probe blocks should have been similar for old and reconfigured chords. However, robust differences were observed between these two chord types, indicating that associations were formed between the elements of the practiced chords. In Experiment 2, we further examined this configural aspect of learning.

In particular, we compared configural responses that involved a single hand (within-hand chords) to those that involved both hands (between-hand chords). Within-hand chords require that novel postures of the hands be produced, whereas between-hand chords may be susceptible to crosstalk between homologous effectors (e.g., Heuer, 1995; Spijkers, Heuer, Steglich, \& Kleinsorge, 2000). These distinct sets of factors may impose different limitations on within- and between-hand chords and thus lead to different types of learning.

To test the role that the two hands play in learning, only two-element chords were used, allowing us to equate the proportion of within-hand and between-hand chords. Because the set of possible chords was small, we did not withhold one of the keys as in Experiment 1. Instead, we focused on the configural aspect of learning in Experiment 2. If learning to overcome bimanual crosstalk contributes to the improvements seen for practiced chords, then bimanual chords should produce larger learning scores than unimanual chords. Alternatively, if learning to make novel postures with the hands played a critical role, then unimanual chords should produce larger learning scores than bimanual chords.

\footnotetext{
${ }^{4}$ An additional ANOVA focused only on the old chords for Trial $\mathrm{n}+1$. The mean RTs for different levels of overlap late in training (Blocks 7 and 8 ) were compared to the mean RTs on probe blocks. Thus, this comparison involved identical sets of chords. Exact repetitions were removed from the analysis because they occurred only during the training blocks. The ANOVA revealed a significant main effect of phase, $F(1,9)=7.38, p<$ .05 , confirming that the RTs for old chords are faster on the final training blocks even when repetitions are removed. However, there was no effect of overlap and no interaction between the two factors, both $F \mathrm{~s}<1$.
} 


\section{Method}

\section{Participants}

Ten participants were recruited from the undergraduate population at the University of California, Berkeley.

\section{Apparatus and Stimuli}

The experimental set-up was identical to Experiment 1 with two exceptions. First, the chord stimuli consisted of just two circles on each trial. Second, a small red circle $(0.36 \mathrm{~cm}$ in diameter) was always visible at the center of the screen. This circle served as a fixation mark and was positioned slightly above the lines at the vertical position of the circles used to signal the chords.

\section{Procedure}

With 10 keys, there are a total of 45 possible two-element chords. We eliminated chords that involved homologous finger responses (or perceptual symmetry), assuming that these might be qualitatively easier than the other pairs. With the elimination of these five chords, the stimulus set consisted of 20 within-hand chords (e.g., both responses made by the left hand or both by the right hand) and 20 between-hand chords (one response by the left hand and one response by the right). The 40 chords were divided into two sets (A and B), each consisting of 10 within-hand chords and 10 between-hand chords with the constraint that each element occurred an equal number of times in each set.

For half of the participants, Set A was used for training; for the other half, Set B was used for training. Participants completed four training blocks consisting of 80 trials each. Thus, at the end of training, each chord stimulus had been presented a total of 16 times. The last two blocks were probe blocks that included two presentations of all 40 chords.

Feedback was provided on trials in which incorrect keys were pressed, target keys were not pressed, or the onset asynchrony of the two key presses was greater than $100 \mathrm{~ms}$. Feedback was also provided at the end of each block indicating both overall accuracy and mean RT.

\section{Results and Discussion}

$R T s$

RTs were computed as the maximum of the two RTs for each of the component responses within a chord. RTs shorter than $250 \mathrm{~ms}$ or longer than $2,500 \mathrm{~ms}$ were eliminated from the analyses $(<1 \%$ of trials). The RTs for each block were submitted to two analyses, one to test the linear trend during the training period and one to evaluate the representational nature of the learning. Although mean RT decreased on each successive block (Figure 3), this effect was not significant in a linear regression restricted to the training phase, $F(1,38)=1.82, p=.19$. The trend was certainly indicative of improvement on this measure: On the first block, participants' mean RT was 1,024 ms; on the fourth block, their mean RT was 914 ms. Although the $110 \mathrm{~ms}$ decrease in RT was not significant, the trend is consistent with learning, and the data from the probe blocks (below) confirm that participants did encode chord-specific learning during training.

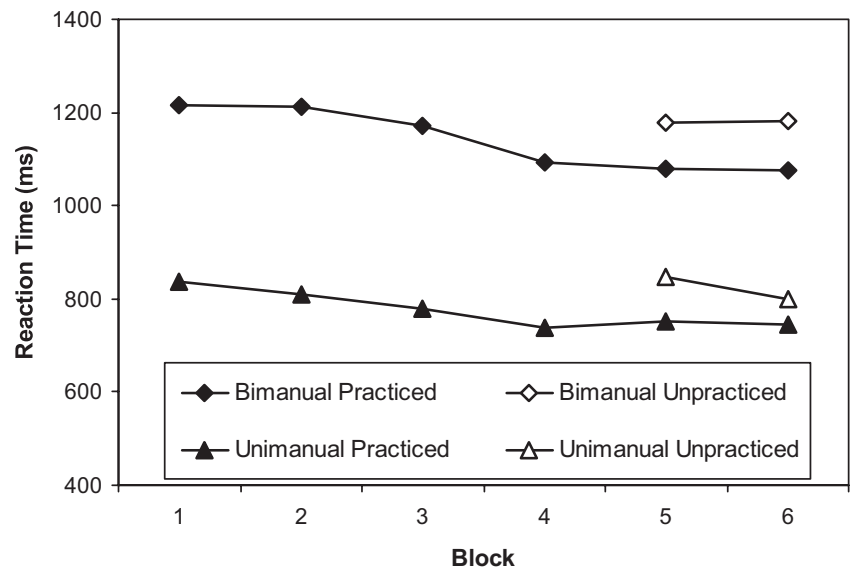

Figure 3. Reaction times in Experiment 2 across the six blocks for the unimanual and bimanual chord types.

Chord learning. Analysis of the data from the probe blocks provides a stronger test of learning. These data were evaluated with a three-way ANOVA, with hand (left, right, or both), chord type (old, reconfigured), and block $(5,6)$ as factors. The main effects of hand, $F(2,18)=194.06, p<.0001$, and chord type, $F(1$, $9)=66.06, p<.0001$, were statistically reliable. The main effect of hand reflected the fact that RTs for the bimanual condition $(1,129 \mathrm{~ms})$ were slower than for the two unimanual conditions, left: $766 \mathrm{~ms}$; right: $805 \mathrm{~ms}$; the difference in RTs for the two hands was not reliable: $t(9)=1.89, p=.09$. Participants were $300 \mathrm{~ms}$ slower when making simultaneous movements with fingers on different hands than with fingers on the same hand. The difference in RT between old and reconfigured chords for the left, right, and bimanual conditions was $50 \mathrm{~ms}, 102 \mathrm{~ms}$, and $101 \mathrm{~ms}$, respectively. The Chord $\times$ Hand interaction was not statistically reliable, $F(2$, 18) $=1.21, p>.3$.

In sum, the results replicate the finding of Experiment 1 that learning is occurring at the configural level. The lack of a difference for the within- and between-hand chords does not support the hypothesis that learning reflects specific processes such as overcoming biases that arise in bimanual coordination. However, this conclusion should be considered cautiously given that it is based on a null result. Nonetheless, the key finding here is that robust configural learning is observed for both within- and between-hand chords.

The unimanual advantage. We were surprised to find that RTs on the bimanual trials were so much slower than on unimanual trials, especially given that configuring one hand to make two movements seems, a priori, more challenging than making a single key press with each hand. One possible account of the difference between uni- and bimanual trials is that the physical distance between the stimuli differs for these two conditions. For example, there are eight unimanual chords, four left and four right, in which the elements are adjacent to each other in the unimanual chord set; in contrast, adjacency is only possible for one bimanual chord, the chord consisting of two thumb responses, and this chord was excluded because it involved homologous movements.

To examine whether the hand effect was related to the distance between the two responses, the data from the probe blocks was 
reanalyzed with a two-way ANOVA in which hand and distance were factors. Distance was defined as the number of fingers between the pair of elements. A distance of 2 corresponded to chords in which the elements were separated by a single stimulus position/finger (e.g., the left ring and index finger, or the left index finger and right thumb). A distance of 4 corresponded to chords in which there were three positions/fingers between the elements (e.g., the left pinky and left thumb, or the left middle finger and right index finger). In order to have a complete factorial design, only trials whose distance ranged between 2 and 4 were included in this ANOVA. No bimanual chords had a distance of 1 and distances greater than 4 were not possible for the unimanual chords. Note that the keyboards for the two hands were positioned by the participants for their own comfort. Thus, we do not mean to suggest that a within-hand distance of 2 is physically equal to a between-hand distance of 2 .

If the cost associated with bimanual responses was solely a function of the physical distance between the stimuli, then the ANOVA should produce a significant effect of distance and no effect of hand. However, the effects of distance, $F(2,18)=8.28, p<.005$, and hand, $F(2$, $18)=159.45, p<.0001$, were both reliable, as was the interaction of these factors, $F(4,36)=8.54, p<.0001$. As shown in Figure 4, the distance factor does not appear to provide a succinct account of the slower responses to bimanual chords. Distance had an effect on RT for the unimanual chords, for which the interkey distance was constant across participants, but RTs were actually fastest when distance was 4 . This is likely due to the fact that this condition requires the combination of the thumb and pinky, the two outer digits and, thus, the least ambiguous stimulus positions. No difference was observed for Distances 2 and 3 for unimanual chords. Similarly, for bimanual chords, distance had no reliable effect on RT. Moreover, the mean RT for bimanual chords with a distance of 2 was considerably longer ( 300 $\mathrm{ms}$ ) than the mean RT for the slowest unimanual condition, the condition with a distance of 3 . In sum, this analysis strongly suggests that the two conditions were qualitatively distinct.

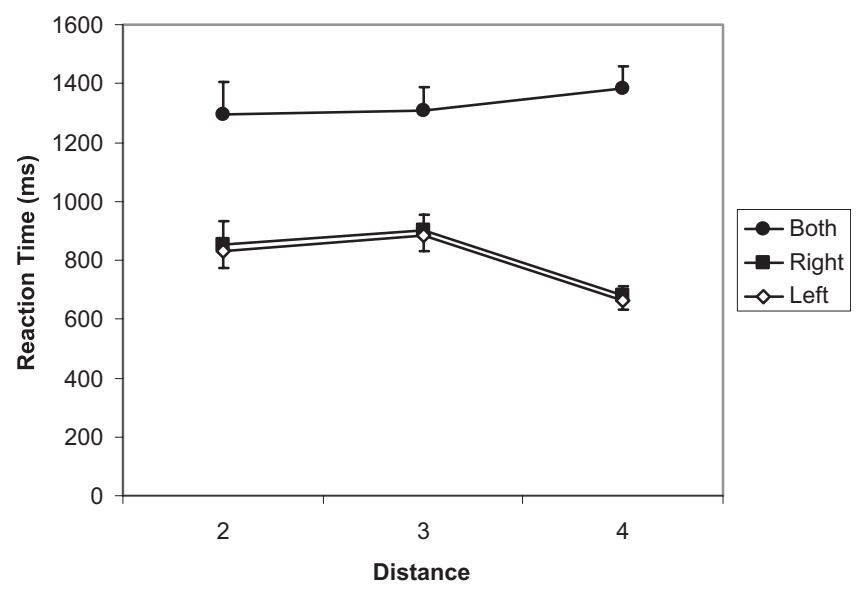

Figure 4. The effects of interkey distance for the three chord types in Experiment 2. Error bars represent 1 standard error of the mean. Distance $=$ the number of fingers between the pair of elements, 2 corresponding to chords in which the elements were separated by a single stimulus position/finger; Both $=$ both hands; Right $=$ right hand and Left $=$ left hand.
Given that the unimanual-bimanual difference cannot be attributed to the distance between the stimuli, the present findings provide preliminary evidence that coordinating simultaneous movements between the two hands requires additional cognitive operations compared to coordinating simultaneous movements within a single hand. For example, the between-hand condition might require coordination of response selection and/or execution operations between the two hemispheres. Several studies using movement precuing tasks (e.g., Adam et al., 1998; Adam \& Pratt, 2004; Miller, 1982, 1985) have reported greater benefits from motor preparation when two responses from a single hand are prepared compared to when two responses from different hands are prepared. This effect may reflect a bias for motor programming to be organized within each of the hands. That is, movements within a hand may be represented more similarly than movements on different hands at some level or levels within the motor system. Such a state of affairs would also benefit the production of unimanual chords relative to bimanual chords. Along these lines, the added cost may reflect the recruitment of neural systems that facilitate bimanual coordination (Kazennikov et al., 1999; Laplane, Talairach, Meininger, Bancaud, \& Orgogozo, 1977).

Unlike Experiment 1, mean RTs to old chords were not slower in the probe blocks. Assuming our strategy hypothesis provides an accurate account of this phenomenon in Experiment 1, the lack of such an effect in Experiment 2 may reflect the fact that the two-finger chord task is easier than the three-finger version. Alternatively, the lack of a cost (and perhaps change in strategy, at least implicitly) may be due to the fact that the new chords all contained practiced elements in Experiment 2.

Sequential effects. As in Experiment 1, we assessed sequential effects by categorizing each trial on the basis of its similarity to the previous trial. We defined three types of sequential conditions: those in which both elements were completely different from the previous trial, those in which one of the elements was repeated, and those in which both elements were repeated. In order to obtain sufficient observations for each subject, the data were collapsed across all six blocks. To avoid confounding the effects of overlap with the effects of the number of hands required to produce the response, only trials involving bimanual responses were analyzed. The difference between partially overlapping chords (993 ms) and nonoverlapping chords $(1,020 \mathrm{~ms})$ was not statistically reliable, $t(9)=1.37, p>.20$. In contrast, RTs in trials involving complete overlap (i.e., trial repetitions) were considerably faster $(792 \mathrm{~ms}$ ) than either the partially overlapping chords, $t(9)=5.19, p<.001$ or nonoverlapping chords, $t(9)=7.69, p<.0001$.

These findings are consistent with Experiment 1; sequential effects appear to be limited to conditions in which the entire chord is repeated. Importantly, these findings demonstrate that, although unimanual chords may be easier to produce than bimanual chords, repetition benefits are obtained only when both hands repeat. Thus, although the postural programming associated with the chords may occur on a hand-by-hand basis, response selection mechanisms appear to operate on representations integrating across the two hands.

\section{Accuracy}

The proportions of correct responses were submitted to ANOVAs identical to those used to analyze the RTs. The one-way ANOVA testing the linear trend in the training data revealed no 
significant increases in accuracy, $F(1,38)=2.36, p=.13$. The ANOVA conducted on the data from the probe phase of the experiment revealed a significant main effect of chord type, $F(1$, $9)=8.60, p<.05$, indicating that old chords were performed more accurately than reconfigured chords (.91 vs. .87 proportion correct). There were no other significant main effects or interactions, all $F$ 's $<1$.

The ANOVA conducted on the training data to evaluate the effects of the distance between the two keypresses revealed a significant main effect of hand, $F(2,18)=4.32, p<.05$, revealing that the left- (.90) and right-hand (.91) chords were responded to more accurately than bimanual chords (.81). Neither the main effect of distance nor the Distance $\times$ Hand interaction approached significance, $F$ 's $<1$. In sum, there was no evidence that a speed-accuracy tradeoff contributed to the patterns of results reported in the RTs.

\section{Experiment 3}

Experiment 1 demonstrated that learning in the chord task is chord specific. Even if each of the individual elements within a chord had been practiced, performance costs were observed when these elements were combined into a chord that had not appeared during training (i.e., reconfigured chords). This finding was replicated in Experiment 2 with chords requiring just two button presses.

An obvious first question is whether the changes following learning are best characterized in terms of perceptual representations or motoric representations. In terms of perceptual learning, participants might become more adept at recognizing the complex patterns formed by the three (Experiment 1) or two (Experiment 2) linear arrays of circles that define the stimulus patterns (Fiser \& Aslin, 2001; Gibson, 1969; Posner \& Keele, 1968). In terms of motor learning, participants might become more facile in producing particular configural responses with their hands. It is also possible that practice leads to both perceptual and motor improvements or improvements at an intermediate level in which particular input patterns are associated with particular chord responses. This latter hypothesis is a form of the S-R translation idea, but now applied to chords rather than the individual elements that form each chord.

We investigated the level of learning in Experiment 3. Participants produced two-element, between-hand chords on all trials. The stimulus for the left hand was always a letter, selected from the set $\mathrm{A}-\mathrm{D}$, presented on the left side of the screen. The S-R mapping for the left-hand responses went in ascending order from left to right (e.g., pinky was A, index finger was D). The stimulus for the right hand could also be a letter, selected from the same set, A-D, or a number, selected from the set 1-4. This stimulus appeared on the right side of the display and indicated which finger should be used with the index finger mapped to the number 1 and letter A, the middle finger to the number 2 and letter B, etc. Note that with this mapping, corresponding letters were assigned to spatially compatible responses (e.g., 'A' requires leftmost responses for both hands) for the two hands rather than anatomically compatible responses (e.g., 'A' requires index finger responses for both hands).

By using two stimulus sets for the right-hand element, each chord could be signaled in two ways, either with two letters or with a letter for the left hand and a number for the right hand. As in Experiments 1 and 2, a subset of the stimuli was not presented in the training blocks whereas all of the stimulus pairs were included in the probe blocks (see Table 1). Of critical interest were two types of transfer probes. For the new-response probes, the two stimuli that mapped to the same response were excluded from the training set (e.g., A3 and AC, both of which are associated with the response left pinky, right ring). For the new-stimulus probes, one member of the pair defining the same chord response was included in the training set and the other was excluded (e.g., A3 included in training but AC excluded, or vice-versa).

Both probes entailed a novel pairing of the two symbolic stimuli. The key difference was that new-response probes required an unpracticed chord response, whereas new-stimulus probes required a chord response that was in the practice set. Performance differences on these two types of probes should indicate the locus of learning in the chord task. If learning is stimulus based, then the new-stimulus and new-response probes should produce similar costs. This pattern is predicted because the individual stimuli in the new-stimulus and new-response probes have been practiced the same number of times, yet the stimulus is novel for both probes. In contrast, if learning is response based, new-stimulus probes should be performed faster than the new-response chords because the former involve response combinations that were practiced during the training blocks.

\section{Method}

\section{Participants}

Sixteen participants were recruited from the undergraduate population at the University of California, Berkeley.

\section{Apparatus and Stimuli}

On each trial, a pair of stimuli, either two letters or a letter and number were used to indicate the chord responses. The left-hand

Table 1

Representative Set of 16 Stimulus Combinations Used on Training Blocks and 8 Stimulus Combinations Added in Probe Blocks in Experiment 3

\begin{tabular}{|c|c|c|c|c|}
\hline \multicolumn{2}{|c|}{ Training stimuli } & \multicolumn{2}{|c|}{ Probe stimuli } & \multirow[b]{2}{*}{ Probe type } \\
\hline Within & Between & Within & Between & \\
\hline $\mathrm{AB}$ & A2 & & & Old \\
\hline \multirow[t]{2}{*}{$\mathrm{AC}$} & A3 & & & Old \\
\hline & & $\mathrm{AD}$ & A4 & New response \\
\hline \multirow[t]{2}{*}{ B1 } & & & BA & New stimulus \\
\hline & $\mathrm{BC}$ & B3 & & New stimulus \\
\hline $\mathrm{BD}$ & B4 & & & Old \\
\hline \multirow[t]{2}{*}{$\mathrm{CA}$} & $\mathrm{C} 1$ & & & Old \\
\hline & & $\mathrm{CB}$ & $\mathrm{C} 2$ & New response \\
\hline $\mathrm{CD}$ & $\mathrm{C} 4$ & & & Old \\
\hline DA & & D1 & & New stimulus \\
\hline \multirow[t]{2}{*}{ DB } & D2 & & & Old \\
\hline & D3 & & DC & New stimulus \\
\hline
\end{tabular}

Note. The actual combinations varied across participants such that the probe stimuli were balanced in terms of the frequency with which each combination was used for old, new stimulus, and new response conditions. Within $=$ within dimension; Between $=$ between dimension. 
element was always signaled by a letter (A, B, C, or D), centered $4.9 \mathrm{~cm}$ to the left of the fixation marker. The same letter set or the numbers 1-4 were used to indicate the right-hand element. This stimulus was centered $4.9 \mathrm{~cm}$ to the right of the fixation marker. The letter-finger mapping for both hands and the digit-finger mapping for the right hand went from left to right in ascending order.

\section{Procedure}

Given that there were only four possible responses for each hand (i.e., the thumbs were not used to make responses in this experiment) and that every trial required a bimanual response, there were just 16 possible configural responses. We excluded chords in which the same letter (e.g., AA) was used for each hand because stimulus repetitions may represent a special case. Thus, there were only a total of 12 possible bimanual chord responses. However, because each right-hand response could be signaled by two different stimuli, a number or a letter, there were 24 possible stimulus combinations.

The training set consisted of 16 of the possible 24 stimulus combinations; the remaining 8 stimulus combinations were also included in the probe set (Table 1). The latter were determined by taking each possible left-hand stimulus, that is, the letters A-D, and choosing one of the possible letters and one of the possible numbers that might be used as a right-hand stimulus. For half of the left-hand letters, the withheld right-hand letter and right-hand number indicated the same response (e.g., $\mathrm{AB}$ and $\mathrm{A} 2$ ). In this case, the letter-letter stimulus combination formed a withindimension, new-response probe and the letter-number stimulus combination formed a between-dimension, new-response probe. For the other half of the left-hand letters, the withheld right-hand letter and right-hand number indicated different responses (e.g., $\mathrm{BC}$ and B4). In this case, the letter-letter stimulus combination formed a within-dimension, new-stimulus probe and the letternumber stimulus combination formed a between-dimension, newstimulus probe. Critically, the other stimulus combination that required the same response combination (e.g., BD for B4) was always present in the training set for the new-stimulus chords. In this way, the combinations of responses were practiced during training even though the new-stimulus probes were not presented. Finally, the withheld right-hand letters were selected so that exactly one of each possible right-hand stimulus (i.e., the letters A-D and the numbers 1-4) was included in the withheld combinations. Therefore, the probability of each possible stimulus was identical across both the training and probe sets.

The timing of each trial was as in Experiments 1 and 2 with the exception that the intertrial interval was reduced to 1,000 ms. Each of the 16 training chords was presented six times in each training block for a total of 96 trials per block. Each probe block consisted of 4 instances of all 24 possible chords, 16 of which were old chords, 4 of which were new-stimulus chords, and 4 of which were new-response chords.

\section{Results and Discussion}

\section{$R T s$}

RTs were again computed as the maximum of the two RTs for each of the component elements within a chord. RTs less than 250 ms or greater than $2,500 \mathrm{~ms}$ were eliminated from the analyses $(<$ $6 \%$ of trials). As in the previous experiments, RTs were submitted to two analyses, one to test the linear trend in RTs during the training period and one to evaluate the representational nature of the learning. The linear regression conducted on the data from the training phase of the experiment revealed a significant decrease in RTs across the six training blocks, $F(1,190)=54.88, p<.0001$, although inspection of the RTs suggests that the decreases were larger earlier in training than late in training (Figure 5).

Chord learning. The data from the probe blocks was submitted to a two-way ANOVA, with dimension (same vs. different) and chord type (old, new-stimulus, and new-response) as factors. Both main effects were significant, dimension, $F(1,15)=8.73$, $p<.01$, chord type, $F(2,30)=15.60, p<.0001$, but their interaction was not, $F<1$ (Figure 6). The main effect of dimension reflected the fact that responses were faster for the different dimension trials $(1,147 \mathrm{~ms})$ than for the same dimension trials $(1,181 \mathrm{~ms})$. Two possible accounts of this effect are plausible. First, it may be that participants found it easier to encode the S-R mapping for the number stimuli, and thus RTs were faster when the right hand responded to a number. Second, when both stimuli were letters, participants may have experienced greater interference in selection processes for the two hands given the conceptual overlap of the stimulus codes for the two hands (see Hazeltine, 2005; Hazeltine, Diedrichsen, Kennerley, \& Ivry, 2003; Logan \& Schulkind, 2000). As in Experiment 2, the practiced chords did not show a cost when the probe chords were introduced.

Of greatest interest is the effect of chord type. The old, newstimulus, and new-response chords produced mean RTs of 1,115, 1,152 , and 1,225 ms, respectively. Post hoc comparisons revealed that the difference between the old and new-stimulus chords did not reach significance, $t(15)=1.72, p=.11$, and that RTs for the new-response chords were significantly slower than the old, $t(15)=5.22, p<.0005$, and new-stimulus chords, $t(15)=4.31$,

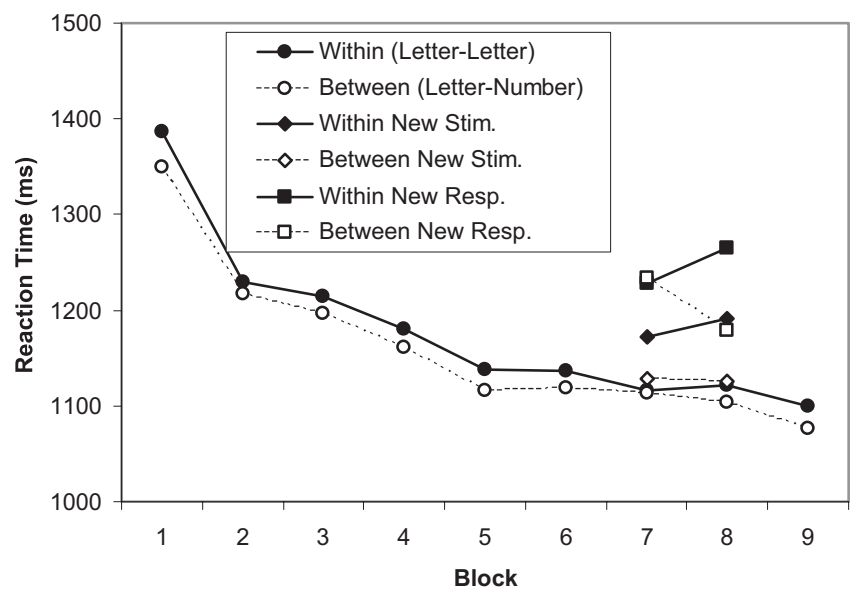

Figure 5. Reaction times in Experiment 3 across the nine blocks for the six chord types. Within trials (i.e., within dimension) included trials in which both stimuli were letters. Between trials (i.e., between dimension) included trials in which the left stimulus was a letter and the right stimulus was a number. New Stim. (new stimulus) trials included trials that required a practiced response to a novel stimulus. New Resp. (new response) trials included trials in which both the stimulus and response were novel. 


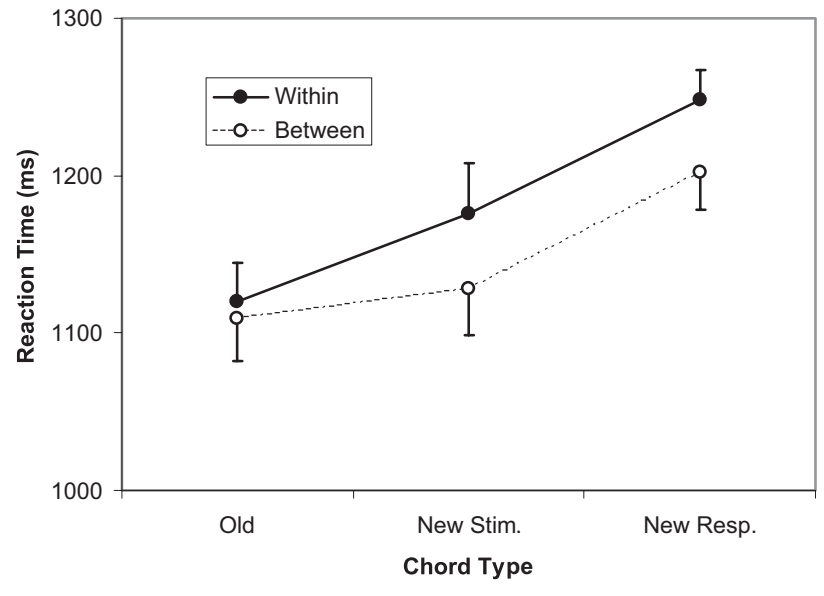

Figure 6. The reaction times during the probe blocks of Experiment 3 for the six chord types. Within $=$ within dimension; Between $=$ between dimension; Stim. $=$ stimulus; Resp. $=$ response.

$p<.001$. These results are consistent with the proposal that learning is primarily response based. That is, transfer was observed such that responses to novel stimuli were faster when these stimuli were mapped to practiced two-element chords compared to when the novel stimuli were mapped to unpracticed two-element chords.

Pure response-based learning? The difference in performance between the new-stimulus and new-response chords indicates that learning was not purely stimulus based. However, the hypothesis that learning is purely response based is sided with the nullhypothesis in this experiment. That is, this hypothesis predicts no difference between the practiced and new-stimulus chords. Although caution is warranted for any conclusion based on null results, this is especially relevant here given that there was a trend for new-stimulus chords to be performed more slowly than the old chords, at least for the within-dimension chords (letter-letter: $1,176 \mathrm{~ms}$ vs. $1,120 \mathrm{~ms} ; t(15)=2.09, p=.053$; letter-number: $1,129 \mathrm{~ms}$ vs. $1,109 \mathrm{~ms} ; t(15)<1)$. Therefore, we cannot rule out the possibility that some stimulus-based learning is occurring, an issue we return to in Experiment $4 .^{5}$ Alternatively, the trend may reflect an intermediate-level representation that includes information from both the stimuli and the responses.

One possible explanation along these lines is that the similarity between the letter and number S-R mappings might include abstract conceptual information relating to the mappings. Given that both the letter- and number-stimulus sets used the same relationship between the ordinal values of the symbols and the spatial locations of the responses (i.e., first symbol mapped to leftmost key, etc.), it is possible that participants encoded the mappings in such a way as to exploit this shared principle (see Hazeltine, 2005). If the chord representations were based partly on these conceptual mapping rules, then the introduction of novel stimulus combinations that invoke the same conceptual rule might have small effects on performance. In this way, a representation that was based on abstract codes would lead to the observed pattern of data in which new-response chords produced larger behavioral costs than newstimulus chords even though the learning was based on S-R rules rather than motor codes.

Sequential effects. As in Experiments 1 and 2, we explored how RTs varied across successive trials. Given the 2:1 mapping used for the right-hand stimuli, exact repetitions of a given stimulus pair were very rare, occurring only about once per participant over the course of the entire session. Therefore, the analysis focused on two forms of repetition: whether the left-hand stimulus repeated (because only one stimulus set was used for the left hand) and whether the right-hand stimulus was the same, excluding the exact stimulus repetitions. We also excluded trials in which the chord response repeated but the right-hand stimulus differed from the right-hand stimulus on the previous trial. A two-way ANOVA was conducted, with dimension of the right-hand stimulus (within vs. between) as one factor and repetition category (left stimulus repeats-right stimulus new, left stimulus new-right stimulus repeats, and both new) as the other factor. In order to obtain sufficient observations for each subject, the data were collapsed across the final six blocks of the experiment.

The main effect of repetition category was statistically reliable, $F(1,15)=54.85, p<.0001$. Post hoc $t$ tests indicated that chord responses were faster for left-hand stimulus repetitions $(1,031 \mathrm{~ms})$ compared to chord responses for right-hand stimulus repetitions (1.161 ms), $t(15)=7.45, p<.0001$, which in turn, were performed more quickly than when both stimuli were new $(1,214 \mathrm{~ms})$, $t(15)=4.20, p<.001$. The main effect of dimension was not reliable, $F(1,15)=2.85, p=.11$, and the two factors did not interact, $F<1$.

In Experiments 1 and 2, repetition benefits were only obtained when the entire stimulus display (and response) was repeated. In contrast, the repetition benefits in Experiment 3 were substantial when only one stimulus repeated (184 ms for the left-hand stimulus; $54 \mathrm{~ms}$ for the right-hand stimulus). Experiment 2 is analogous to the present case, because it also involved two-element chords and the analyses of repetition effects in that experiment were restricted to bimanual trials. The reason for this difference in results between the two experiments requires further investigation. At present, we propose that the inclusion of two distinct mappings for the right hand promoted a conceptualization of the task as including two separate responses. Such a conceptualization may

\footnotetext{
${ }^{5}$ It should be noted that there is a difference between the amount of training associated with the responses for the old- and new-stimulus chords. Whereas all of the stimulus combinations in the practice set are equally probable, responses associated with new-stimulus chords received only half as much practice as the old-stimulus chords in the practice set during training (see Table 1). This is because, for the new-stimulus chords, only one of the two possible stimulus combinations associated with a particular response is presented during training (the other being withheld as the new-stimulus chord). Thus, the trend for RTs for new-stimulus chords to be slower during the probe blocks may be another manifestation of response-based learning. To test this explanation, we compared two types of chords in the practice set. Practiced stimulus combinations involved chord responses shared with the withheld new-stimulus chords (e.g., A3 and $\mathrm{AD}$ during practice when $\mathrm{AC}$ and $\mathrm{A} 4$ were new-stimulus chords) and chords for which both stimulus combinations were included in the practice set (e.g., AB and A2 when they were both old chords). No differences were observed between these two conditions, $t(15)=1.42, p=.18$, despite the fact that the old chords $(1,165 \mathrm{~ms})$ were performed twice as often as chords for which one pair was withheld for the new-stimulus condition $(1,176 \mathrm{~ms})$. Given that the mean RTs for the two types of practiced chords were so similar, the advantage for the new-response chords compared to the newstimulus chords in the probe blocks does not appear to be based entirely on practice of the motoric component of the responses.
} 
have been reinforced by the fact that all of the trials required one response for each hand. In other words, the task demands may have encouraged participants to treat the responses for the two hands as more independent than when the responses were signaled by a nearly continuous array of spatial stimuli. Despite this difference, the magnitude of the benefit of practiced chords compared to new-chord responses was similar across the two experiments (101 ms in Experiment 2 and $113 \mathrm{~ms}$ in Experiment 3).

\section{Accuracy}

The proportions of correct responses were submitted to ANOVAs identical to those used to analyze the RTs. For the ANOVA evaluating the effects of dimension and chord type, neither main effects nor the interaction was statistically reliable. There was a trend for new-response chords (.87 proportion correct) to be performed less accurately than practiced chords (.92). These results are largely consistent with the findings from the RT data.

For the sequential effects, the ANOVA produced significant main effects of both dimension, within: .88 proportion correct, between: $.90, F(1,15)=5.15, p<.05$, and repetition category, both new stimuli: .87 , right repeated stimulus, .89 , left repeated stimulus, $.91 ; F(1,15)=7.76, p<.005$. Thus, pattern of accuracies is highly similar to the pattern of RTs, indicating that the results do not stem from a speed-accuracy tradeoff.

\section{Experiment 4}

In Experiment 3, participants were faster in responding to novel stimuli that mapped to practiced responses compared to novel stimuli that mapped to unpracticed responses. However, there are two potential objections to the conclusion that this result indicates that learning in the first two experiments was largely response based. First, the chord responses were signaled by symbolic stimuli rather than the spatially compatible stimuli used in Experiments 1 and 2. Cueing the responses symbolically may have increased the complexity of S-R translation processes and added a conceptual component to the task that was amenable to the benefits of learning. In other words, the use of the symbolic cues may have enabled participants to encode relationships between the stimulus categories, a relationship not present in Experiments 1 and 2. Second, as noted previously, it remains unclear whether there is also some stimulus-based learning. Experiment 3 did not test whether RTs are faster to previously observed stimuli compared to novel stimuli, a more direct test of stimulus-based learning.

Experiment 4 was designed to address both of these concerns. On each trial, a stimulus display consisted of two spatial stimuli as in Experiments 1 and 2. On chord task blocks, participants again pressed two keys simultaneously, making configural responses based on the positions of the stimuli. To examine stimulus-based learning, we designed a new task, the neighbor task, which required participants to carefully analyze the perceptual structure of the stimuli but not to make configural responses. For this task, participants compared the positions of the two stimulus elements and, with a single keypress, indicated whether the elements occupied neighboring positions.

The two tasks were performed in alternating blocks throughout the experiment. For the practice blocks, the stimuli were divided into four sets. One set was presented during practice of the chord task only and a second set was presented during practice of the neighbor task only. A third set was presented during practice with both tasks. The fourth set was not presented during practice for either task. In the final probe blocks for each task, all of the stimuli from the four sets were presented with equal probability. The critical question was whether the benefits of practice would be task specific. In particular, to test stimulus-based learning, we focused on whether exposure to the stimuli in the neighbor task would be sufficient to produce learning when these same stimuli were tested in the chord task.

We added one additional manipulation in this experiment. Given that the chord task requires learning novel manual configurations, we included a postexperimental questionnaire to assay the amount of musical training for each participant. This information was used to evaluate the effects, if any, of prior musical experience, keyboard experience in particular, on measures of configural performance and learning.

\section{Method}

\section{Participants}

Thirteen participants were recruited from the undergraduate population at the University of California, Berkeley. The data from one participant was not used in the analyses because of high error rates on the neighbor task.

\section{Apparatus and Stimuli}

Throughout the experiment, a row of eight horizontal lines and a fixation cross were displayed on the computer monitor. The lines were grouped into four lines on the left of the fixation cross and four lines on the right of the cross. The four lines corresponded to the four fingers of each hand. Each line was $1.1 \mathrm{~cm}$ in length and distance between lines within a side was $2.2 \mathrm{~cm}$. The distance between the two groups of lines was $4.9 \mathrm{~cm}$. On each trial, a two-element chord was presented by replacing two of the dashes by $\mathrm{Xs}$, one on each side of the fixation cross.

\section{Procedure}

Participants were instructed that they would be performing two tasks in alternating blocks. For the chord task, each of the horizontal lines was mapped to one of eight keys on the two keyboards in a spatially compatible fashion as in Experiment 2. Participants were instructed to make both keypresses simultaneously and to do so as quickly as possible. The keypresses were made with the four fingers of each hand; the thumbs were not used.

For the neighbor task, participants were instructed to judge whether the two Xs would be neighbors if they were presented on the same row of dashes. For example, if the element on the left side was in the second position, then the participant would classify the stimulus as a neighbor if the element on the right side of the display was in the first or third position. If the element on the right side was in the second or fourth position, the correct response would be to indicate that the two elements were not neighbors. Note that identical positions (e.g., both elements in second position within their respective cluster of four lines) did not count as neighbors. "Wrap-around" was allowed so that positions 1 and 4 were considered neighbors. In this way, there were an equal 
number of neighbor and nonneighbor displays, and each position was uncorrelated with a particular response.

Given four possible positions for the left element and four for the right element, there were 16 possible displays. To ensure that differences among the chords (for the chord task) did not determine the pattern of results, the 16 stimuli were divided into four sets, with each set consisting of one spatially compatible chord (e.g., ring finger of left hand and middle finger of right hand), one anatomically compatible chord (e.g., ring finger of left hand and ring finger of right hand), one compatible chord for which the two elements were neighbors, and one incompatible chord for which the two elements were not neighbors. Thus, half the chords were neighbors and half were not. Moreover, each of the eight possible element positions was occupied exactly once across the four chords in each of the four sets.

The four sets were used to make four training ensembles. A training ensemble consisted of the 16 stimulus patterns divided into the four sets: patterns that appeared during training for both the chord and neighbor task (shared), patterns that appeared during training only for the chord task (chord only), patterns that appeared during training only for the neighbor task (neighbor only), and patterns that did not appear during training at all (probe). The assignments of the four sets were counterbalanced across the four training ensembles so that each set served as each chord type (e.g., shared, chord only, etc.) across the four ensembles. Therefore, for each participant, the trial types were balanced in terms of the probability of the $\mathrm{X}$ appearing in each stimulus position; the probability of being spatially compatible, anatomically compatible, or incompatible; and the correct response for the neighbor task. Across participants, each possible stimulus served as each chord type an equal number of times.

Participants completed 14 blocks of the experiment, each with 64 trials. On odd-numbered blocks, they performed the chord task, making simultaneous keypresses to the two stimuli. On even-numbered blocks, they performed the neighbor task, using the index and middle finger of the right hand to press either the $V$ or $B$ key on the computer keyboard for neighbor and nonneighbor responses, respectively. The first 10 blocks were training blocks in which each of the eight training chords (four shared and four chord only on chord-task blocks and four shared and four neighbor only on neighbor-task blocks) was presented eight times. The final four blocks were probe blocks in which each of the 16 possible chords was presented four times. The timing of each trial was as in Experiment 3.

After completing the experiment, participants responded to a questionnaire to determine the extent of their musical training. The questionnaire asked them to list the musical instruments they had playing throughout their lives and to report how much practice that had with each instrument. Based on their responses, the participants were divided into four categories: 0 , those responding that they had no musical experience; 1, those responding that they played a nonkeyboard instrument at a beginner level; 2, those with 3 or fewer years playing a piano; and 3 , those with more than 3 years of practice on a piano.

\section{Results and Discussion}

\section{$R T s$}

RTs on the chord task were again computed as the maximum of the two RTs for each of the component elements. For the neighbor task, only a single finger press was made on each trial. For both tasks, RTs shorter than $250 \mathrm{~ms}$ or longer than 3,000 ms were eliminated from the analyses $(<4 \%$ of trials), but the tasks were analyzed separately. As in the previous experiments, RTs were submitted to a linear regression to test the linear trend in RT during the training blocks and an ANOVA to evaluate transfer of chord knowledge during the probe blocks. The linear regression revealed a significant linear decrease in RTs across the training blocks for both the chord task, $F(1,118)=8.05, p<.005$, and the neighbor task, $F(1,118)=16.59, p<.0001$ (see Figure 7). For the chord task, RTs decreased $178 \mathrm{~ms}(1,268 \mathrm{~ms}$ on Block 1 vs. 1,090 ms on Block 9); for the neighbor task, RTs decreased $398 \mathrm{~ms}(1,642 \mathrm{~ms}$ on Block 2 vs. 1,424 ms on Block 10).

An additional ANOVA tested whether the two types of practice stimuli showed the same benefits during practice. The stimuli that were practiced using both tasks did not differ significantly from those that were practiced only with one task in either the chord, $F(1,11)<1$, or the neighbor task, $F(1,11)=2.90, p=.12$. Furthermore, this factor did not interact with block for either task: chord task, $F(4,44)=1.51, p=.22$; neighbor task, $F(4,44)<1$. In short, during training, there was no evidence that exposure to a particular stimulus during the performance of one task improved performance with that stimulus on the other task.

Transfer in the chord task. To assess what information was encoded during training, the data from the transfer blocks for the chord task were submitted to a three-way ANOVA, with block (11 vs. 13), chord exposure (new vs. old), and neighbor exposure (new vs. old) as factors. Only the main effect of chord exposure was statistically reliable, $F(1,11)=16.12, p<.005$. The main effect of neighbor exposure approached significance, $F(1,11)=4.71$, $p=.053$. No other main effects or interactions approached significance, all $F$ 's $<1.72$; all $p$ 's $>$.2. Practiced chord responses were performed $95 \mathrm{~ms}$ faster than unpracticed responses. In contrast, the advantage for seeing a stimulus in the neighbor task was $35 \mathrm{~ms}$ over stimuli that had not been seen during the neighbor task. The greater advantage for practicing the chord compared to observing the stimulus in the neighbor task was significant, $t(11)=$ $2.19, p=.05 .^{6}$

These results indicate that performance in the chord task is primarily driven by practicing the particular chord responses rather than exposure to the particular stimuli. Like the chord task, the neighbor task required participants to analyze the configural properties of the stimulus. Indeed, RTs for the neighbor task were significantly longer in the neighbor task than in the chord task, $t(11)=305, p<.0001, M=257 \mathrm{~ms}$, suggesting that the smaller benefit for chord performance did not result from participants attending to the stimuli less during the neighbor task. Despite spending more time processing the stimuli during the neighbor task, participants showed greater benefit from practicing stimuli with the chord task. This conclusion is reinforced by the data from the practice blocks, in which exposure to the stimuli in both tasks provided no additional benefits to performance in either task.

Taken together, Experiments 3 and 4 provide complementary evidence that learning in the chord is largely response based. Experiment 3 demonstrated that novel stimuli requiring practiced

\footnotetext{
${ }^{6}$ This $t$ test is equivalent to a test comparing the chord-only probes to the neighbor-only probes.
} 

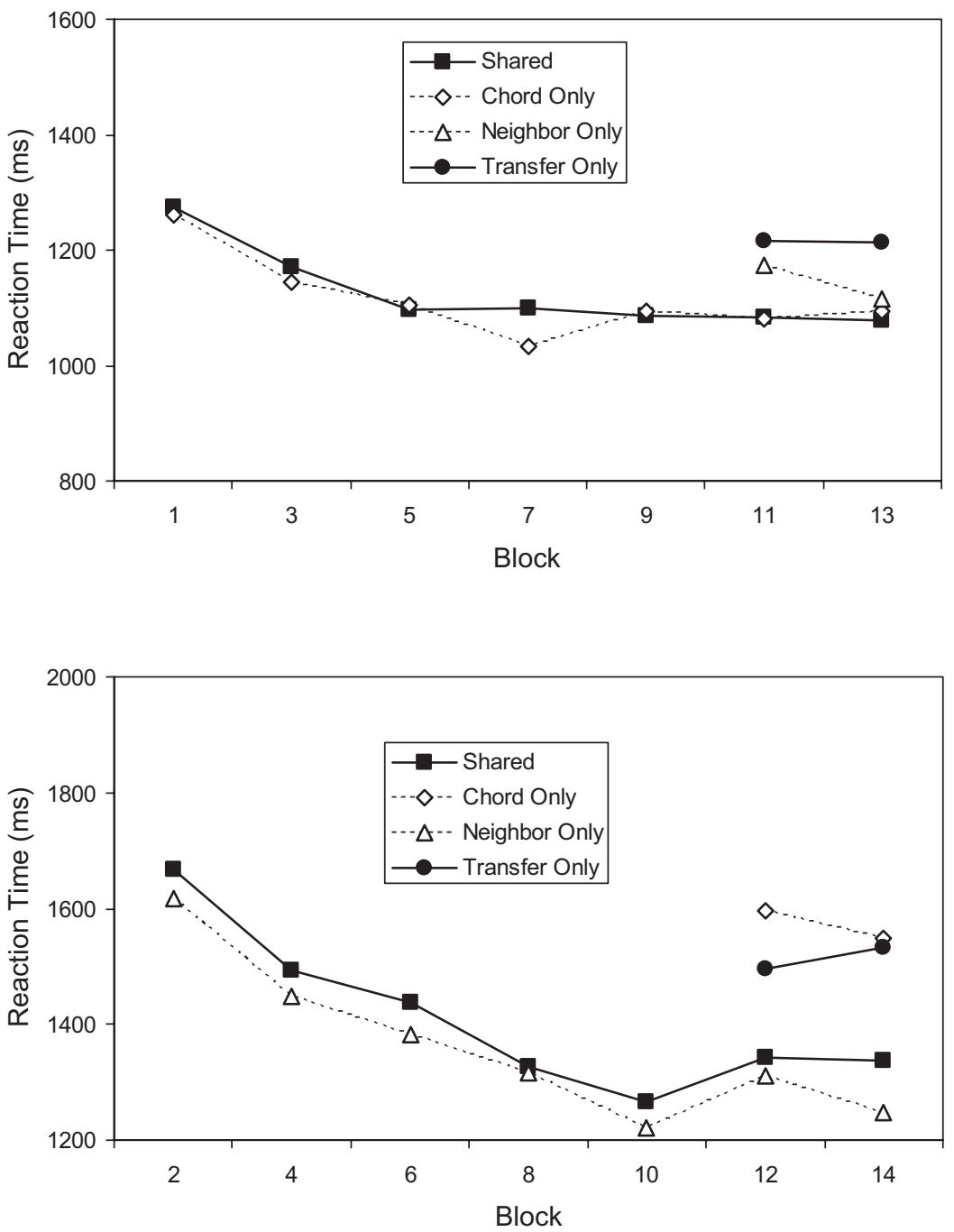

Figure 7. Reaction times in Experiment 4 across the 14 blocks task (top) and the neighbor task (bottom) for the four chord types.

chord responses were performed faster than novel stimuli requiring unpracticed chord responses. Experiment 4 demonstrated that familiar stimuli with practiced chord responses were performed faster than familiar stimuli with unpracticed chord responses. Moreover, unlike the stimuli in Experiment 3, the stimuli in Experiment 4 did not involve abstract categories that may have served as a medium for transfer.

Transfer in the neighbor task. The RTs from blocks 12 and 14 (when participants performed the neighbor task) were also submitted to a three-way ANOVA. Only the main effect of neighbor exposure was statistically reliable, $F(1,11)=27.38, p<.001$. The main effect of chord exposure approached significance, $F(1,11)=$ $4.24, p=.064$ No other main effects or interactions approached significance, all $F$ 's $<1.53$, all $p$ 's $>$.2. As with the chord task, exposure to the stimuli in the same task led to an advantage in performance, with RTs to the stimuli that were practiced in the neighbor task $234 \mathrm{~ms}$ faster than those that had not been practiced in the neighbor task. In contrast, exposure to the stimuli for the chord task, if it had any effect, impaired performance on the neighbor task (RTs to stimuli to which participants had practiced chord responses were $59 \mathrm{~ms}$ slower than to stimuli to which they had not). In sum, whereas there was a nonsignificant trend suggesting the exposure to a particular stimulus in the neighbor task benefited performance in the chord task, there was no evidence that performing a particular stimulus in the chord task benefited performance in the neighbor task.

Sequential effects. Sequential effects were evaluated as in Experiment 2. In order to obtain sufficient observations for each subject, the data were collapsed across the final eight blocks of training. For the chord task, the difference between partially overlapping chords $(1,125 \mathrm{~ms})$ and nonoverlapping chords $(1,107 \mathrm{~ms})$ was not statistically reliable, $t(11)<1$. In contrast, trials involving complete overlap (i.e., trial repetitions) were considerably faster (907 ms) than either the partially overlapping chords, $t(11)=4.17$, $p<.001$, or nonoverlapping chords, $t(11)=3.15, p<.01$. These findings were consistent with the other experiments. Sequential 
effects appeared to be limited to conditions in which the entire chord is repeated.

The neighbor task showed a nearly identical pattern. The difference between partially overlapping chords (1,343 ms) and nonoverlapping chords $(1,381 \mathrm{~ms})$ was not statistically reliable, $t(11)=1.80, p>$.1. Trials involving complete overlap (i.e., trial repetitions) were considerably faster $(943 \mathrm{~ms})$ than either the partially overlapping chords, $t(11)=4.91, p<.001$, or nonoverlapping chords, $t(11)=5.52, p<.001$.

Musical training. The mean RTs from the final two practice blocks and the learning scores (computed as the difference between practiced and unpracticed stimuli) were correlated with musical experience scores for both tasks. For the chord task, there was a significant correlation between RT and musical training, $r=$ $-.69, t(10)=-2.62, p<.05$. However, there was no indication that the level of musical training affected the amount of learning, $r=.10, t<1$. The pattern of correlations was very similar for the neighbor task. Musical training was correlated with RT, $r=-.70$, $t(10)=-2.74, p<.05$, but not learning, $r=-.22, t<1$. Thus, the advantage observed in terms of overall speed on the chord task for those with more extensive musical training does not appear to necessarily be motoric, or at least specific to configural responses, given that a similar correlation is found for the one-key responses on the neighbor task. Moreover, in both tasks, there was little evidence that musical training affected learning.

\section{Accuracy}

The proportions of correct responses were submitted to ANOVAs identical to those used to analyze the RTs. The ANOVA conducted on the data from the probe phase of the experiment revealed no significant main effects of chord exposure, $F(1,11)=$ $2.33, p=.16$, or neighbor exposure, $F<1$, nor did the two factors interact, $F<1$.

For the analysis of sequential effects, the accuracies showed the same pattern as the RTs. The complete-overlap chords were performed more accurately than either the nonoverlapping or partialoverlap chords for both tasks: chord task, complete (1.00) vs. nonoverlapping (.92), $t(11)=8.20, p<.0001$, complete vs. partial (.93): $t(11)=6.84, p<.0001$; neighbor task, complete (.98) vs. nonoverlapping (.92): $t(11)=2.52, p<.05$; complete vs. partial (.94), $t(11)=2.32, p<.05$. However, there were no differences between the partial and nonoverlapping chords for either task: chord task, $t<1$; neighbor task, $t(11)=1.45, p=.17$. In short, there was no evidence of a speed-accuracy tradeoff underlying the effects observed in the RTs.

\section{General Discussion}

We modified the classic experiment of Seibel (1963) to study configural response learning, an aspect of motor control that has received relatively little attention. Unlike commonly studied tasks, such as the SRT task or force field learning where learning requires the acquisition of predictions of upcoming sensorimotor events, the chord task requires that the participant learn to efficiently adopt a range of hand configurations. This nonpredictive aspect of coordination is an important component of many skills. Learning appropriate hand postures is essential for skills as diverse as tennis and manipulating chopsticks, in addition to the hand configurations learned by pianists to produce chords on the keyboard. Behavioral evidence suggests that postures play a privileged role in the representation of perceived movements (Marteniuk \& Roy, 1972), and neurophysiological evidence indicates that postures play a critical role in the representation of produced movement (Graziano, Taylor, \& Moore, 2002).

\section{How Do We Learn to Produce Chords?}

Unlike sequence learning, the chord-learning task requires that multiple effectors be coarticulated rather than activated separately across distinct points in time. Importantly, learning in the chord task is based on this requirement of coactivation; the configural properties of the chords were encoded, not just the individual elements. In each of the four experiments, a significant cost was observed for novel chords composed of individual elements that had been practiced in other configurations during training. Thus, improvements in performance must relate to the relationships among the individual elements. In this sense, configural response learning can be described as the learning of novel postures, albeit restricted to configurations of the hands.

Configural responses were clearly encoded, but there was also evidence for learning at the element level. In Experiment 1, one of the digits was not used at all during practice. During the probe blocks, novel chords involving the withheld digit were produced more slowly than either old chords or reconfigured chords. This pattern suggests that some learning occurs at the element level in addition to that associated with the relationships among the elements.

However, there remains an alternative explanation for elementlevel learning. It is possible that the advantage for the reconfigured probes compared to the new-finger probes results from greater similarity between the reconfigured and practiced chords. That is, the reconfigured chords overlap with the practiced chords to a greater degree than the new-finger chords overlap with the practiced chords. It may be that the greater overall similarity between the trained and transfer stimuli, in terms of shared elements, is responsible for the advantage of reconfigured chords over novel chords rather than the lack of exposure to the withheld stimulus per se. As such, it is possible that all learning occurred at the configural level rather than at the element level. This question is a topic for further research.

Along these lines, it is important to note that the same learning process may underlie the development of configural associations and the encoding of the S-R mappings for individual elements. Using a computational model, Gupta and Cohen (2002) demonstrated that a single learning mechanism could account for performance benefits associated with familiar stimuli and performance benefits observed when novel stimuli are used for the same task. Extended to the current study, performance costs associated with the novel and reconfigured costs in Experiment 1 could reflect the same learning process.

Although our data do not speak directly to the issue of whether separate mechanisms encode the individual S-R associations and the configural properties of the chords, they do constrain the types of information encoded during learning. Because the reconfigured chords are performed more slowly than the practiced ones, we conclude that the learning involves the encoding of configural information about the chords. That is, the learned representation 
must specify relationships between particular keypresses. This is true even when the chords consist of just two fingers and the two fingers belong to distinct hands. In fact, in Experiment 2, the magnitude of the learning benefit for separate hands was similar to the magnitude of the benefit for within-hand chords. Thus, improvements in the chord task are not simply the result of learning to rapidly adopt novel hand gestures. A more apt description is that the participants learned to produce configural responses-that is, they learned to produce novel postures.

The results of Experiments 3 and 4 indicate that learning is largely response based. In Experiment 3, we compared performance on two types of chords: chords composed of new combinations of stimuli that required unpracticed responses and chords composed of new combinations of stimuli that required practiced responses. Participants responded faster on trials in which the stimulus was new but required a practiced response compared to trials in which the stimulus was new and required a new response. In Experiment 4, we employed a control task in which the participants were exposed to the same stimulus displays as in the chord task, but only a single keypress was required rather than configural responses. When chord responses were required to all of the displays, practiced chords were performed faster than those that had not been practiced, arguing against a stimulus-based account of learning. Together, the two experiments provide converging evidence that learning in the chord task includes the encoding of configural responses.

This is not to say learning by observation (e.g., Mattar \& Gribble, 2005) is not possible in this task. It may be that individuals can learn to make the configural responses by watching others produce them. However, the findings suggest that the actions/ postures are a critical component of the learned representation. Whether the actual production of these postures is essential for learning is a topic for further research.

The finding that configural learning in the current experiments is primarily response based is somewhat unexpected given the findings of Hazeltine et al. (2002). In that study, participants were as adept in producing speeded manual and vocal responses to novel stimulus pairings as they were to highly practiced pairings, suggesting a lack of integrated, or configural, learning. There are several possible reasons for the different pattern of results between these studies. Unlike the current chord-learning task, the stimuli and responses for the two tasks in Hazeltine et al. involved distinct modalities. This may reduce the likelihood of forming associations between the two responses. Moreover, there was no requirement that the two responses be produced at the same time. Thus, in the Hazeltine et al. study, the participants likely conceptualized the experiment as involving two distinct tasks, whereas in the current chording task, the displays and responses would favor an integrated representation.

In both Experiments 3 and 4, there was some evidence for stimulus-based learning, but in neither case was this effect statistically reliable. These trends, if they reflect actual differences in performance, may reflect perceptual learning. For example, pattern recognition mechanisms might become sensitive to familiar arrays of the stimulus elements (e.g., Fiser \& Aslin, 2001; Gabrieli et al., 1990; Gibson, 1969; Posner \& Keele, 1968). Alternatively, the trends may stem from the fact that some aspects of learning are neither purely stimulus based nor purely response based. Several researchers have proposed that motor programming may take place at an intermediate level between sensory and motor processing (Erlhagen \& Schöner, 2002; Gopher et al., 1985; Hazeltine, 2002, 2005; Hommel, Müsseler, Aschersleben, \& Prinz, 2001; Miller, 1982).

This point was made eloquently in Seibel's (1963) study, in which participants showed little change in RT after practice when comparing conditions involving 31 and 1,024 response alternatives. Learning to perceive the stimuli or produce the chords more quickly does not account for why RT was minimally affected by the number of response alternatives. Consistent with Seibel's interpretation, we assume that learning can strengthen the links between stimuli and their associated responses, that is, by making response selection more efficient. However, unlike Seibel's interpretation, we propose that learning is based on representations of whole chords rather than individual S-R elements.

\section{How Are Chords Represented by Response Selection Processes?}

The sequential analyses for Experiments 1, 2, and 4 provide further support for the hypothesis that learning in the chord task arises at the configural level. In the first two experiments, there was little behavioral benefit for chords that partially overlapped with the chord on the previous trial. In contrast, exact repetitions yielded reductions in RTs on the order of hundreds of milliseconds. Therefore, the chords appear to be coded as a unitary response by selection mechanisms. This pattern is consistent with the chord-specific learning observed in all four experiments.

It is possible that sequential effects for partially overlapping chords entailed a trade-off between a (partial) repetition benefit and a cost associated with reconfiguring the elements from one chord to the next. Hommel and colleagues have proposed that the production of a response involves a binding operation in which the various features of the response are bound into an action file (Hommel, 2004; Hommel et al., 2001). When subsequent actions overlap in terms of these features, the features must be unbound with the original action file before they can be combined into a new action file. This additional operation can result in a cost in RT (Hommel, 2004; Stoet \& Hommel, 1999). If such an operation influenced sequential effects in the present experiment, one might expect an advantage on trials in which the required chord response had no shared elements with the previous chord. Although complex trade-offs are possible, an analysis in Experiment 1 based on element overlap failed to support this hypothesis.

The pattern of repetition effects was considerably different for Experiment 3, in which partially overlapping chords did benefit performance on immediately successive trials by more than 100 $\mathrm{ms}$. This difference may reflect the more complex S-R mappings used in this experiment. Alternatively, the asymmetrical roles of the two hands during the task may play a critical role. The different $\mathrm{S}-\mathrm{R}$ mappings for the two hands may encourage participants to encode the two responses as belonging to separate tasks.

Despite differences in the way that the bimanual responses may have been conceptualized (e.g., as two tasks instead of one), chord learning was robust and similar in magnitude to that observed in Experiment 2. It is possible that one aspect of learning is to link the responses regardless of whether or not they involve unitary representations at the level of response selection processes. That is, practice may establish links between particular keypresses, and 
these links may facilitate the coactivation of the appropriate responses during chord performance. This process of coactivation could produce behavioral benefits when the two responses are conceptualized as components of separate tasks (as appears to be the case in Experiment 3) or when the two responses form an integrated whole (as appears to be the case in Experiments 1, 2, and 4). In this way, advantages for practiced compared to unpracticed chords may reflect processes distinct from those revealed by sequential effects.

\section{How Does Chord Learning Relate to Other Forms of Motor Learning?}

Chord learning occurred without the participants being aware that our experimental manipulation included the introduction of novel chords during the probe blocks. This is similar to what has been reported in studies using the SRT task, in which learning can develop in the absence of awareness of the sequence. Thus, participants can demonstrate their acquired skill in the chord and SRT tasks without being able to verbally express their knowledge, much like real world motor skills.

A notable difference, of course, between the chord and SRT tasks is that the latter requires developing associations between successive events whereas, for chord learning, the components of the response must be executed simultaneously. Together, configural and sequential learning capture two important aspects of skilled actions. It is possible that the distinction between these two forms of learning is superficial, with similar associative mechanisms operating either at a common point in time (configural learning) or across time (sequential learning).

However, computational considerations suggest that these forms of learning may serve different purposes. Theoretical accounts of learning in the SRT task focus on associations between temporally distinct events. In some cases, these associations are assumed to be links between responses (e.g., Willingham, 1999; Willingham et al., 2000); others have proposed that links are formed between a response and the subsequent stimulus (e.g., Ziessler \& Nattkemper, 2001, 2002). Nonetheless, a common theme in these accounts is that learning serves to generate expectations about upcoming events. It is difficult to explain how associations between motor commands and subsequent sensory events or subsequent motor commands can account for chord learning, at least in the present context. Of course, one could modify the task such that the perceptual consequences of the chord responses provide an important source of feedback; for example, the resulting sounds likely provide a salient cue to reinforce chord learning for the novice at the piano.

We do not intend to argue that the learning mechanisms engaged by the chord-learning task are necessarily distinct from those engaged by the SRT task. Despite the different task demands, it is an open question whether learning is achieved by overlapping sets of processes for the two tasks. Neuroimaging and neuropsychological studies are likely to prove useful in exploring this issue, given the extensive literatures that exist concerning the neural bases for sequence learning. Already, some neuroimaging studies have identified networks of brain structures engaged during the observation of complex movements relating to playing a musical instrument (Buccino et al., 2004; Haslinger et al., 2005), although these studies have emphasized the imitative aspects of the move- ments rather than the encoding of particular movement configurations. By expanding our set of model tasks for studying motor learning, we can acquire a richer picture of skill acquisition.

\section{References}

Adam, J. J., Paas, F. G. W. C., Teeken, J. C., van Loon, E. M., van Boxtel, M. P. J., Houx, P. J., et al. (1998). Effects of age on performance in a finger-precuing task. Journal of Experimental Psychology: Human Perception and Performance, 24, 870-883.

Adam, J. J., \& Pratt, J. (2004). Dissociating visual attention and effector selection in spatial precuing tasks. Journal of Experimental Psychology: Human Perception and Performance, 30, 1092-1106.

Amazeen, P. G. (2002). Is dynamics the content of a generalized motor program for rhythmic interlimb coordination? Journal of Motor Behavior, 34, 233-251

Ammons, R. B., Ammons, C. H., \& Morgan, R. L. (1958). Subskills in rotary pursuit as affected by rate and accuracy requirements and by distribution of practice. Journal of General Psychology, 58, 259-279.

Buccino, G., Vogt, S., Ritzl, A., Fink, G. R., Zilles, K., Freund, H-J., et al. (2004). Neural circuits underlying imitation learning of hand actions: An event-related fMRI study. Neuron, 42, 323-334.

Cohen, A., Ivry, R. B., \& Keele, S. W. (1990). Attention and structure in sequence learning. Journal of Experimental Psychology: Learning, Memory, and Cognition, 16, 17-30.

Cohen, N. J., \& Squire, L. R. (1980). Preserved learning and retention of pattern-analyzing skill in amnesia: Dissociation of knowing how and knowing that. Science, 210, 207-210.

Curran, T., \& Keele, S. W. (1993). Attention and nonattentional forms of sequence learning. Journal of Experimental Psychology: Learning, Memory, and Cognition, 19, 189-202.

Erlhagen, W., \& Schöner, G. (2002). Dynamic field theory of movement preparation. Psychological Review, 109, 545-572.

Fiser, J., \& Aslin, R. N. (2001). Unsupervised statistical learning of higher-order spatial structures from visual scenes. Psychological Science, 12, 499-504.

Gabrieli, J. D. E., Milberg, W., Keane, M. M., \& Corkin, S. (1990). Intact priming of patterns despite impaired memory. Neuropsychologia, 28, 417-427.

Gibson, E. J. (1969). Principles of perceptual learning and development. New York: Appleton-Century-Crofts.

Gopher, D., Karis, D., \& Koenig, W. (1985). The representation of movement schemas in long-term memory: Lessons from the acquisition of a transcription skill. Acta Psychologia, 60, 105-134.

Graziano, M. S. A., Taylor, C. S. R., \& Moore, T. (2002). Complex movement evoked by microstimulation of precentral cortex. Neuron, 34, 841-851.

Gupta, P., \& Cohen, N. J. (2002). Theoretical and computational analysis of skill learning, repetition priming, and procedural memory. Psychological Review, 109, 401-448.

Haslinger, B., Erhard, P., Altenmüller, E., Schroeder, U., Boecker, H., \& Ceballos-Baumann, A. O. (2005). Transmodal sensorimotor networks during action observation in professional pianists. Journal of Cognitive Neuroscience, 17, 282-293.

Hazeltine, E. (2002). The representational nature of sequence learning: Evidence for goal-based codes. In W. Prinz \& B. Hommel (Eds.), Attention and Performance (Vol. XIX, pp. 673-689). Oxford, England: Oxford University Press.

Hazeltine, E. (2005). Response-response compatibility during bimanual movements: Evidence for the conceptual coding of action. Psychonomic Bulletin \& Review, 12, 682-688.

Hazeltine, E., Diedrichsen, J., Kennerley, S., \& Ivry, R. B. (2003). Bimanual cross-talk during reaching movements is primarily related to response selection, not the specification of motor parameters. Psychological Research, 67, 56-70. 
Hazeltine, E., Teague, D., \& Ivry, R. B. (2002). Simultaneous dual-task performance reveals parallel response selection after practice. Journal of Experimental Psychology: Human Perception and Performance, 28(3), $527-545$.

Heuer, H. (1995). Models for response-response compatibility: The effects of the relation between responses in a choice task. Acta Psychologia, 90, $315-332$.

Hick, W. E. (1952). On the rate of gain of information. Quarterly Journal of Experimental Psychology, 4, 11-26.

Hommel, B. (2004). Event files: Feature binding in and across perception and action. Trends in Cognitive Science, 8, 494-500.

Hommel, B., Müsseler, J., Aschersleben, G., \& Prinz, W. (2001). The theory of event coding (TEC). Behavioral and Brain Sciences, 24, $849-878$

Hyman, R. (1953). Stimulus information as a determinant of reaction time. Journal of Experimental Psychology, 45, 188-196.

Kazennikov, O., Hyland, B., Corboz, M., Babalian, A., Rouiller, E. M., \& Wiesendanger, M. (1999). Neural activity of supplementary and primary motor areas in monkeys and its relation to bimanual and unimanual movement sequences. Neuroscience, 89, 661-674.

Keele, S. W., Ivry, R. B., Mayr, U., Hazeltine, E., \& Heuer, H. (2003). The cognitive and neural architecture of sequence representation. Psychological Review, 110, 316-339.

Keele, S. W., Jennings, P., Jones, S., Caulton, D., \& Cohen, A. (1995). On the modularity of sequence representation. Journal of Motor Behavior, 27, 17-30.

Kirsner, K., \& Speelman, C. (1996). Skill acquisition and repetition priming: One principle, many processes? Journal of Experimental Psychology: Learning, Memory, and Cognition, 22, 563-575.

Laplane, D., Talairach, J., Meininger, V., Bancaud, J., \& Orgogozo, J. M. (1977). Clinical consequences of corticectomies involving the supplementary motor area in man. Journal of Neurological Science, 34, 301314

Logan, G. D. (1990). Repetition priming and automaticity: Common underlying mechanisms? Cognitive Psychology, 22, 1-35.

Logan, G. D., \& Schulkind, M. D. (2000). Parallel memory retrieval in dual-task-situations: I. Semantic memory. Journal of Experimental Psychology: Human Perception and Performance, 26, 1072-1090.

MacKay, D. G. (1982). The problem of flexibility, fluency, and speedaccuracy trade-off in skilled behavior. Psychological Review, 89, 483506.

Marteniuk, R. G., \& Roy, E. A. (1972). The codability of kinesthetic location and distance information. Acta Psychologica, 36, 471-479.

Martin, T. A., Keating, J. G., Goodkin, H. P., Bastian, A. J., \& Thach, W. T. (1996). Throwing while looking through prisms. I. Focal olivocerebellar lesions impair adaptation. Brain, 119, 1183-1198.

Mattar, A. A. G., \& Gribble, P. L. (2005). Motor learning by observing. Neuron, 46, 153-160.

Miller, J. (1982). Discrete versus continuous models of human information processing: In search of partial output. Journal of Experimental Psychology: Human Perception and Performance, 8, 273-296.

Miller, J. (1985). A hand advantage in preparation of simple keypress responses: Reply to Reeve and Proctor (1984). Journal of Experimental Psychology: Human Perception and Performance, 11, 221-233.

Newell, K. M., Liu, Y-T., \& Mayer-Kress, G. (2001). Time scales in motor learning and development. Psychological Review, 108, 57-82.

Nissen, M. J., \& Bullemer, P. (1987). Attentional requirements of learning: Evidence from performance measures. Cognitive Psychology, 19, 1-32.

Poldrack, R. A., \& Gabrieli, J. D. E. (2001). Characterizing the neural mechanisms of skill learning and repetition priming: Evidence from mirror reading. Brain, 124, 67-82.

Poldrack, R. A., Selco, S. A., Field, J. E., \& Cohen, N. J. (1999). The relationship between skill learning and repetition priming: Experimental and computational analyses. Journal of Experimental Psychology: Learning, Memory, and Cognition, 25, 208-235.

Posner, M. I., \& Keele, S. W. (1968). On the genesis of abstract ideas. Journal of Experimental Psychology, 77, 353-363.

Rabbitt, P. M. A., Fearnley, S., \& Vyas, S. M. (1975). Programming sequences of complex responses. In P. M. A. Rabbitt \& S. Dornic (Eds.), Attention and performance (Vol. V, pp. 295-317). London: Academic Press.

Ratz, H. C., \& Ritchie, D. K. (1961). Operator performance on a chord keyboard. Journal of Applied Psychology, 45, 303-308.

Schwartz, B. L., \& Hashtroudi, S. (1991). Priming is independent of skill learning. Journal of Experimental Psychology: Learning, Memory, and Cognition, 17, 1177-1187.

Seibel, R. (1963). Discrimination reaction time for a 1,023-alternative task Journal of Experimental Psychology, 66, 215-226.

Shadmehr, R., \& Mussa-Ivaldi, F. A. (1994). Adaptive representation of dynamics during learning of a motor task. Journal of Neuroscience, 14 $3208-3224$

Spijkers, W., Heuer, H., Steglich, C., \& Kleinsorge, T. (2000). Specification of movement amplitude for the left and right hands: Evidence for transient parametric coupling from overlapping-task performance. Journal of Experimental Psychology: Human Perception and Performance, 26, 1091-1105.

Stoet, G., \& Hommel, B. (1999). Action planning and the temporal binding of response codes. Journal of Experimental Psychology: Human Perception and Performance, 25, 1625-1640.

Ungerleider, L. G., Doyon, J., \& Karni, A. (2002). Imaging brain plasticity during motor skill learning. Neurobiology of Learning and Memory, 78, 553-564.

Van Selst, M., Ruthruff, E., \& Johnston, J. C. (1999). Can practice effects eliminate the psychological refractory period effect? Journal of Experimental Psychology: Human Perception and Performance, 25, 1268 1283.

Welch, R. B. (1978). Perceptual modification: Adapting to altered sensory environments. New York: Academic Press.

Willingham, D. B. (1998). A neuropsychological theory of motor skill learning. Psychological Review, 105, 558-584.

Willingham, D. B. (1999). The neural basis of motor-skill learning. Current Directions in Psychological Science, 8, 178-182.

Willingham, D. B., Wells, L. A., Farrell, J. M., \& Stemwedel, M. E. (2000). Implicit motor sequence learning is represented in response locations. Memory and Cognition, 28, 366-375.

Zanone, P. G., \& Kelso, J. A. S. (1997). Coordination dynamics of learning and transfer: Collective and component levels. Journal of Experimental Psychology: Human Perception and Performance, 23, 1454-1480.

Ziessler, M., \& Nattkemper, D. (2001). Learning of event sequences is based on response effect learning: Further evidence from serial reaction task. Journal of Experimental Psychology: Learning, Memory, and Cognition, 27, 595-613.

Ziessler, M., \& Nattkemper, D. (2002). Effect anticipation in action planning. In W. Prinz \& B. Hommel (Eds.), Attention and Performance (Vol. XIX, pp. 645-672). Oxford, England: Oxford University Press.

Received February 25, 2005

Revision received January 26, 2006

Accepted January 28, 2006 